\title{
De la nécessité d'un inventaire forestier écologique
}

par

Jean-Louis Brown ${ }^{1}$

\section{Résumé}

Afin de satisfaire nos besoins croissant en matière ligneuse il faudra investir plusieurs millions de dollars dans l'aménagement forestier, sans pour autant avoir un succès assuré si nous n'avons pas une connaissance valable des écosystèmes forestiers. On propose ici une approche simple pour réaliser une carte forestière "écologique". Le territoire est stratifié en fonction des milieux physiques qui montrent des différences pertinentes pour la croissance de la forêt. A l'intérieur d'une région écologique donnée, les habitats qui peuvent se traduire principalement par le type de dépôt, la classe de drainage, la pente, la situation topographique...., sont cartographiés avec les peuplements actuels qu'ils supportent. Une telle approche permet une classification et une cartographie rapides des différents écosystèmes forestiers. Par la suite, la détermination des séries évolutives peut se faire rapidement en reliant entre eux les différents stades de la végétation inventoriés sur des habitats similaires. On obtient ainsi une image dynamique de la forêt permettant de prévoir son évolution. De même la cartographie intégrée de la végétation forestière et du milieu physique permet de relier les résultats des différents traitements sylvicoles aux facteurs du milieu et de les extrapoler aux habitats analogues.

Les coûts supplémentaires pour ajouter à la carte forestière les données relatives à I'habitat sont vite amortis par les erreurs coûteuses d'aménagement qu'elles permettent d'éviter. De plus, l'exercice que requiert la détermination de l'habitat augmente la précision de l'interprétation des peuplements. Le coût est aussi amorti par le fait que les contours de base de la carte correspondent à une assise naturelle stable et sont donc permanents. Seul l'état de la végétation devra périodiquement être remis à jour.

\section{Abstract}

To meet our growing needs for wood products will require several million dollars of investment in forest management without assured success, unless we have an appropriate knowledge of forest ecosystems.

This paper proposes a simple approach to laying down an "ecological" forest map. Physical habitats which can be related to landform, position on the slope, drainage, etc., within an ecological region, are mapped with their actual stands. This approach leads to a rapid classification and mapping of the different forest ecosystems. It is then possible to work out different chronological sequences or seres by relating the stands occupying similar habitats. Knowledge of seres and of actual stand allows the manager to foresee the evolution of a particular stand. In the same way, by the integrated mapping of forest stands and physical habitats, results of sylvicultural treatments can be related to habitat factors, and subsequently can be applied to similar ecosystems.

Added costs of mapping data relative to habitats are rapidly compensated by avoiding costly management errors. Furthermore, the supplementary exercise of determining the habitat increases the precision of photo interpretation for stand mapping. The cost is also compensated by the fact that map contours are permanent since they are based on natural and stable features. Only vegetation data will have to be brought up to date periodically.
Au Québec, jusqu'à tout récemment, il ne s'est fait que des efforts timides et souvent isolés d'aménagement forestier. Nous misions sur l'abondance de la ressource qu'on disait inépuisable. Aussi s'est-on borné le plus souvent à un aménagement de récolte en présumant que la forêt se régénérerait aussi rapidement que nous pouvions la couper. Avec nos besoins sans cesse grandissant pour le bois d'oeuvre et la fibre, nous sommes devenus de plus en plus ingénieux pour récolter rapidement et économiquement le

Service de la recherche, (Terres et Forêts), Ministère de l'Énergie et des Ressources du Québec. bois. Cependant les travaux pour régénérer la forêt et stimuler sa croissance ont progressé bien pius modestement. Comme le déclarait J. Thompson au $73^{e}$ congrès de l'A.C.I.B., "nous avons surexploité nos ressources forestières". De sorte qu'à ce rythme, nous allons aujourd'hui rapidement vers une pénurie de bois. Et, avec la crise de l'énergie, on peut présumer facilement que le rythme d'exploitation de la ressource ligneuse ira en s'accélérant.

II devient donc urgent de s'orienter vers un aménagement de plus en plus intensif de la forêt. Cela implique des investissements importants. Mais, il ne faudrait pas procéder aveuglément et gaspiller inutilement temps et argent. Pour éviter 
cela, il faut se donner des outils et le premier pas en ce sens est la confection d'une carte forestière "écologique", c'est-àdire une carte montrant à la fois la végétation forestière et le milieu qu'elle colonise. Une telle carte permet non seulement de connaître l'état actuel de la forêt, mais également, en reliant la végétation aux différents milieux qu'elle colonise, de classifier sur une base pratique quant à la croissance des essences, les différents milieux quel que soit l'état de la forêt ou même en l'absence de celle-ci. En bref, elle rend possible les travaux d'aménagement en harmonie avec les facteurs du milieu, maximisant ainsi nos chances de succès.

Les pertes monétaires découlant d'erreurs d'aménagement dues à la méconnaissance écologique des milieux traités peuvent se chiffrer en millions de dollars. Par exemple, Overend (1981) relate que par des erreurs de site dans le reboisement en Colombie Britannique, une plantation de Douglas de 30 ans s'est arrêtée de croître. Elle devra être détruite et le site replanté avec une essence appropriée. Cela triple les dépenses de reboisement et occasionne une perte de 30 ans de production pour ce site. Quelques sites ont dû être ainsi replantés jusqu'à 5 fois. Le succès de la régénération naturelle après coupe est relié lui aussi en grande partie aux facteurs du milieu et aux modes de récolte, comme le souligne Doucet (1981).

Ces constatations illustrent bien qu'en foresterie, il faut être doublement prudent dans les choix d'aménagement et se donner pour cela toute l'information nécessaire pour minimiser les risques d'erreurs. Car, contrairement à ce qui peut se produire en agriculture, où l'échec d'une récolte se constate au cours d'une même saison de croissance, une erreur dans le choix d'essences en forêt peut causer la perte de plusieurs décennies de croissance. Ainsi, les pertes occasionnées par la destruction d'une variété de blé d'Inde très productive, par des conditions climatiques défavorables se répétant tous les 20-25 ans, sont minimes par rapport à celles résultant de la destruction par le même phénomène d'une plantation de 20 ans d'une essence non acclimatée.

Pour les productions agricoles, on peut investir des sommes assez considérables afin d'améliorer le sol et le remettre en état à la suite de différentes cultures, car on en retire un rendement annuel et il faut bien continuer de se nourrir. En foresterie, est-on prêt à faire un tel aménagement intensif? Nous ne travaillons pas à le même échelle et il faut être plus prudent pour protèger les sols forestiers et éviter leur dégradation.

\section{Type de carte proposée:}

On peut rêver d'une carte écologique très sophistiquée, donnant une foule de détails. Cependant, nous avons déjà un urgent besoin d'un document construit selon une logique uniforme pour tout le Québec méridional. Un premier document qui se voudrait trop élaboré serait à ce moment-ci non seulement trop coûteux pour l'usage qu'on peut en faire dans les premières phases d'aménagement, mais aussi et surtout trop long à produire. Aussi faut-il, dans une première étape, penser à un document qui soit le plus simple possible, se restreignant aux données les plus pertinentes et répondant aux besoins immédiats ou à ceux du "futur rapproché" des aménagistes. Ultérieurement, il sera toujours possible de perfectionner le document lorsque des modes d'aménagement de plus en plus intensif nécessiteront des informations complémentaires

II ne faut donc retenir, dans un premier temps, que les paramètres qui traduisent le mieux un ensemble de facteurs du milieu pertinents à la croissance de la forêt; paramètres qu'il devrait être possible de cartographier rapidement à l'aide de la photo aérienne.
L'essentiel de la méthode de classification et de cartographie de la végétation forestière proposée ci-dessous s'appuie principalement sur un précédent travail (Brown 1981a) qui portait sur l'étude détaillée d'écosystèmes forestiers et la mise en relief des caractéristiques, tant écologiques que photographiques, utiles ou nécessaires à leur discrimination sur la photo aérienne. Cette méthode repose donc sur la notion d'écosystème, c'est-à-dire qu'elle considère que la végétation forme un tout avec le milieu physique qu'elle occupe et dont elle reflète la fertilité

Les conditions du milieu variant depuis le pied des collines jusqu'à leur sommet, cela se traduit, selon ce même gradient, par une succession de groupements différents d'essences, qui forment ainsi des séquences topographiques particulières selon les différentes régions écologiques. À titre d'exemple, dans la forêt du Témiscamingue (Brown 1981a), lorsque les collines sont moyennement élevées, leur sommet arrondi et leur pente régulière, on observe depuis le pied des collines vers leur sommet, la séquence de végétation suivante (figure 1): la bétulaie jaune à sapin sur le till profond ou délavé, moyennement à imparfaitement drainé, du fond de la vallée: l'érablière à bouleau jaune typique, var. à érable à épis, sur le till profond moyennement drainé, sur le bas versant concave; l'érablière à bouleau jaune typique sur le till plus ou moins profond (à mince), bien drainé, du moyen et du haut versant; enfin, l'érablière à ostryer et chêne rouge sur le till mince et bien drainé du sommet.

Cette séquence illustre bien les changements dans le couvert végétal climacique selon différents habitats, tels que traduits par la position sur la pente, la nature et l'épaisseur du dépôt et la classe de drainage. Ces principaux caractères de I'habitat s'interprètent bien sur la photo. Ils ne changent que peu ou pas dans le temps, contrairement à la végétation. Cette dernière peut s'éloigner plus ou moins du climax par suite de sa modification ou même de sa destruction par des agents naturels (feux, chablis, épidémies) ou à la suite de l'intervention humaine (coupes partielles ou totales). La séquence topographique simple (c'est-à-dire donnant le stade ultime de

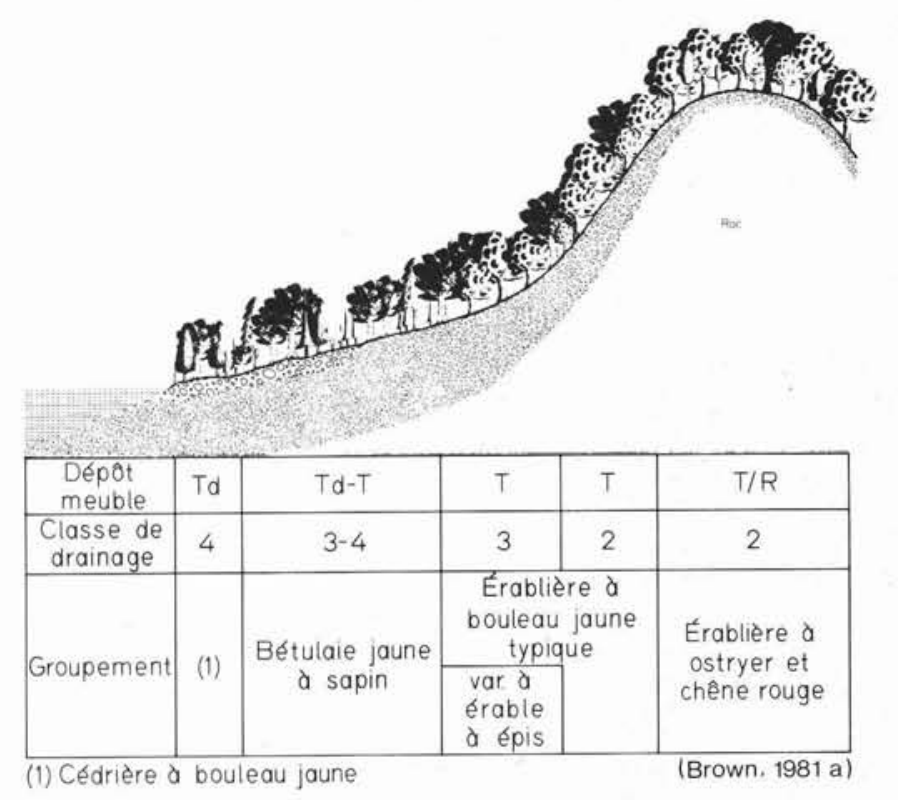

Figure 1. Répartition en fonction de la position sur la pente, du dépôt et de la classe de drainage des principaux peuplements climaciques du sous-domaine septentrional de l'érablière à bouleau jaune. 


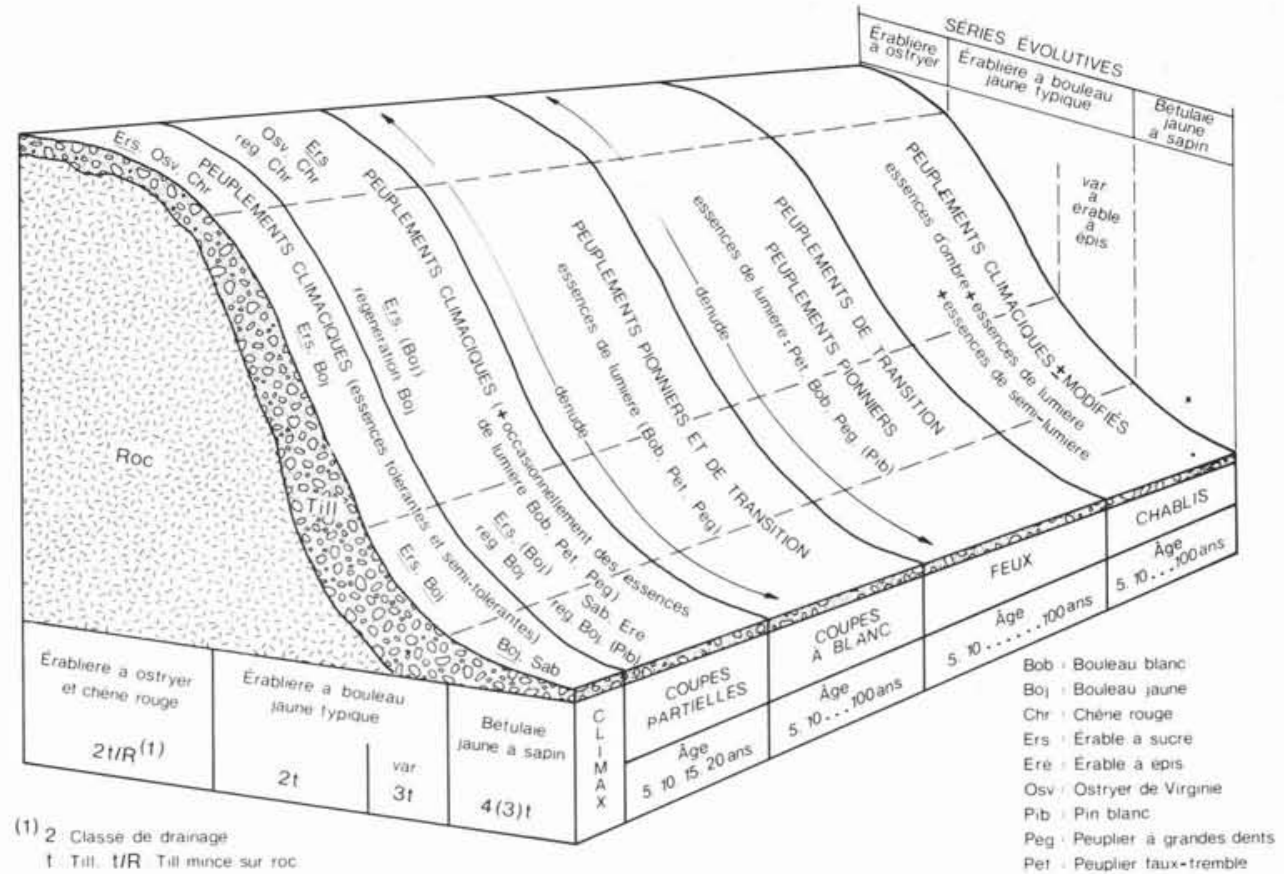

Figure 2. Illustration de l'enchaînement des différents groupements d'essences des séries évolutives au moyen des caractéristiques de l'habitat (position sur la pente, dépôt meuble, classe de drainage).

la végétation en équilibre avec le climat régional et les autres facteurs du milieu) donnée en exemple ci-dessus, peut donc se compliquer davantage à la suite de catastrophes naturelles ou de traitements anthropiques qui permettent à des essences de lumière (peupliers, bouleau blanc, pins) de s'imbriquer dans ces peuplements climaciques ou de constituer des peuplements pionniers ou de transitions qui s'intercalent dans cette séquence, voire même remplacent complètement les groupements climaciques (figure 2).

Selon la spécificité des milieux, les évènements naturels qui s'y sont déjà produits ou les traitements auxquels on a soumis la forêt ou le milieu forestier, on aura donc une végétation différente quant à sa composition, sa structure et sa productivité. Elle réagira aussi différemment aux traitements auxquels elle sera soumise et demandera un mode d'aménagement particulier.

Avec les ressources dont nous disposons actuellement, nous pouvons difficilement définir et caractériser dans les détails chacun des stades de la végétation, mais nous pouvons les regrouper rapidement et les situer, par le biais de leur habitat, à l'intérieur de séries évolutives et en arriver ainsi à prévoir leur évolution. C'est pourquoi nous proposons, pour cette première étape, de cartographier les essences avec leur habitat, ce dernier étant définit en fonction de 1) sa situation sur la pente, 2) du type de dépôt, 3) de sa composition minéralogique et de 4) la classe de drainage (avec ou sans "seepage"). Les caractéristiques no 1, 2 et 4 se voient ou s'interprètent bien sur la photo tandis que la caractéristique nO 3 peut se déterminer à partir de l'étude de cartes géologiques complétée par des observations sur le terrain et l'interprétation photographique de l'assise rocheuse.

Pour chacun des habitats ainsi cartographiés, il s'agit d'ajouter le groupement d'essence qui le colonise en nommant les essences dominantes, identifiées directement par leur image photographique ou déduites par photointerprétation. Les autres renseignements pertinents pour l'aménagiste tels que la pente (classe), l'âge du peuplement (classe), la structure, etc. devraient également être cartographiés.

L'échelle de la carte devra être assez grande pour permettre de distinguer les différents milieux physiques pertinents pour la croissance de la forêt. En général au Québec, une échelle de l'ordre 1/20 000 ou plus grande permet une cartographie suffisamment détaillée de la plupart des écosystèmes forestiers, pour répondre aux besoins actuels des aménagistes.

\section{Quelques applications de l'inventaires forestier écologique}

La classification des forêts en fonction du milieu physique répond aux besoins les plus souvent exprimés par les aménagistes. Comme nous l'avons déjà souligné (Brown 1979 1981 b), étant basée sur un ensemble de facteurs pertinents à la croissance de la forêt, elle fournit une assise stable qu permet, pour chacune des essences, la confection de tables de rendement plus précises et permanentes. Elle facilite et rationnalise la comptabilité forestière. II existe aujourd'hui des lecteurs de carte, couplés avec des ordinateurs qui les rendent capables de lire des contours sur une carte, de mémoriser leur contenu et de tracer à leur tour des cartes. En tenant à jour une telle banque de données, on est en mesure de connaître et de localiser sur la carte, presque à chaque instant, l'état de la forêt, le volume et l'accroissement de chacune des essences qu'elle renferme en fonction de la fertilité du milieu, ainsi que la localisation et la superficie des peuplements qui la constituent.

Une telle classification des forêts assure aussi une meilleure compréhension des expériences passées. Les effets des différents traitements sur la forêt et le milieu peuvent être mieux suivis et analysés en fonction des différents facteurs en présence. Les nouvelles connaissances ainsi acquises pourront être rapidement extrapolées aux autres peuplements semblables croissant dans des milieux analogues. 


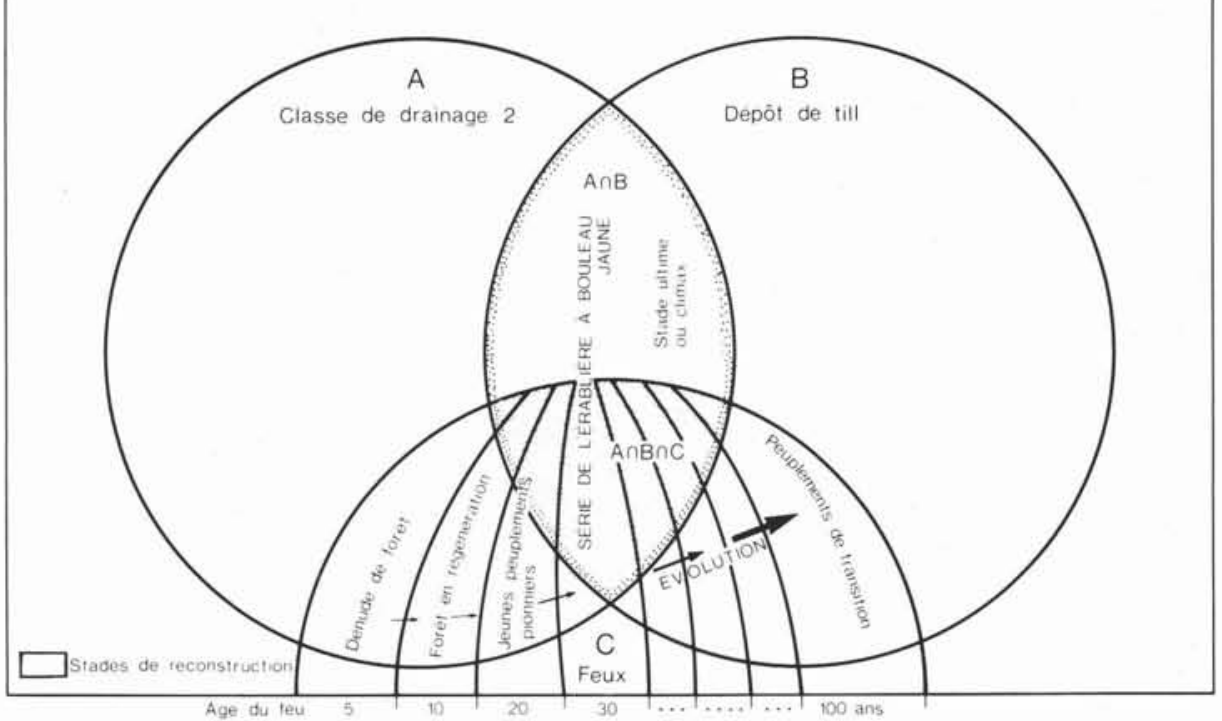

Figure 3. Diagramme illustrant la discrimination et le classement des écosystèmes de la série évolutive de l'érablière à bouleau jaune, sur till bien drainé (classe de drainage 2), pour réaliser une étude dynamique de la recolonisation de tel habitat à la suite de la destruction du couvert forestier par le feu, et ensuite prédire l'évolution future de la végétation sur des habitats analogues dans 5 ans, 10 ans, ... 100 ans à la suite d'une semblable perturbation.

La planification de la recherche bénéficiera également d'un cadre écologique pour s'orienter d'abord vers les écosystèmes les plus répandus et les plus importants. Les expériences s'appuieront sur une meilleure connaissance des écosystèmes étudiés, maximisant ainsi dès le départ les chances de succès et facilitant par la suite l'analyse et la compréhension des résultats obtenus. L'application de ceuxci sera rationalisée et grandement facilitée car on pourra les extrapoler facilement aux écosystèmes ou aux milieux analogues.

Enfin, un tel inventaire constitue un outil quasi indispensable, vu la grandeur du territoire québécois, pour réaliser rapidement des études de dynamisme. L'aménagiste se pose constamment les questions suivantes en regard de différents peuplements qu'il doit aménager: Qu'arrivera-t-il à la suite d'un chablis ou d'un feu dans tel type de peuplement dans 5 ans, 10 ans, 20 ans, etc.? Quel sera dans $X$ années l'état de la forêt ou le peuplement de remplacement à la suite de telle intervention? Quel sera le rendement du prochain peuplement, le délai de régénération? Doit-on laisser faire la nature ou intervenir et quand? Autant de questions auxquelles il sera souvent possible de répondre promptement, si nous avons déjà inventorié, pour des habitats similaires, l'état actuel de la végétation ayant déjà subi ces divers traitements ou résultant de ceux-ci. Car une fois que l'information d'un tel inventaire est emmagasinée sur bande magnétique, il ne s'agit alors que de faire, à l'aide de l'ordinateur, les recoupements nécessaires de manière à discriminer un ensemble de conditions très précises et étudier ensuite l'état actuel de la végétation (figure 3).

Comme l'illustre la figure 2, il suffirait de bien définir le type d'habitat (c'est-à-dire la région écologique, la position sur la pente, le type de dépôt, la classe de drainage) du peuplement dont on veut connaître l'origine ou l'évolution et d'étudier les différents stades de végétation actuellement rencontrés sur le terrain. Par exemple, dans tout le domaine climacique de l'érablière à bouleau jaune, il existe sans doute plusieurs stations d'érablière à bouleau jaune qui ont été dévastées par le feu à différentes époques dans le passé (figure 2 et 3 ). Aujourd'hui, si l'on veut connaitre ce qui adviendra dans 10 ans, 20 ans, 30 ans ...., 100 ans, à la suite de la destruction par le feu d'une telle érablière, on peut s'en faire une bonne idée en étudiant l'état actuel de la végétation qui croît sur des habitats d'érablières à bouleau jaune qui ont déjà été détruites par le feu il y a également 10 ans, 20 ans, 30 ans ..., 100 ans.

Le même raisonnement s'applique pour les chablis et les différents types de coupe. De telles études peuvent nous aider à connaître l'évolution de la végétation à la suite de ces traitements et nous indiquer si l'on doit intervenir et à quelle époque précise, pour hâter cette évolution ou l'orienter vers la satisfaction de besoins très précis.

En bref, la carte forestière écologique fournit non seulement l'image actuelle de la forêt avec les données nécessaires à la récolte, mais montre également une image dynamique de celle-ci, rendant possible un véritable aménagement basé sur des données pertinentes.

Malgré le surplus d'informations qu'elles apporte, son coût en regard de celui d'une simple carte de peuplement n'est pas nécessairement exorbitant. Car pour un interprête qualifié, le temps requis pour ajouter la nature du dépôt, la classe de drainage et le terme de la série évolutive n'est souvent pas beaucoup plus long que le temps nécessaire à leur inscription sur la photo. De plus, le coût supplémentaire d'un tel document peut être amorti assez rapidement si l'on considère que les contours des milieux physiques sont permanents; il n'y aura que l'état de la végétation qui changera dans le temps. II ne s'agira donc, par la suite, que de mettre à jour périodiquement, à l'intérieur de chaque contour, l'état de la forêt à la suite de son évolution lente ou de changements draconiens produits par des agents naturels (feux, épidémies, chablis ...) ou des actions anthropiques (coupes, plantations ....). L'utilisation de données écologiques pour la photo-interprétation des peuplements ou des groupements d'essences, en plus de critères purement photographiques, facilite grandement la photo-interprétation et augmente la précision de la cartographie. 
La carte forestière écologique est un document essentiel pour l'aménagement des forêts et des terrains forestiers. Le temps et l'argent qu'elle permettra d'économiser en évitant des erreurs coûteuses justifient amplement le coût supplémentaire de sa production, comparativement à celui d'une simple carte forestière.

\section{Remerciements}

L'auteur remercie Messieurs Yvon Richard, René Doucet, Léon Carrier, Dennis Walsh et Fabien Caron du Ministère de l'Énergie et des Ressources pour leurs commentaires et $L$. Beaulieu pour le dessin des illustrations.

\section{Références}

Brown, J.-L., 1979. Méthode écologique d'inventaire forestier. Rapport pour le Comité de classification écologique du Service de l'inventaire forestier. Service de la recherche forestière. Min des Terres et Forêts du Québec. $5 p$

Brown, J.-L., 1981a. Les forêts du Témiscamingue, Québec. Écologie et photo-interprétation. Étude écologique n0 5. Laboratoire d'écologie forestière, Univ. Laval, Québec. 447 p. 20 stéréogrammes $\mathrm{N}+\mathrm{B}, 16 \mathrm{pl}$. couleur h.t.

Brown, J.-L., 1981b. L'écologie, un outil d'aménagement. Conférence présentée au $61^{\mathrm{e}}$ congrès annuel de l'Ordre des ingénieurs forestiers du Québec. In Forêt, moteur de développement. O.I.F.Q. p. 53-63

Doucet, R., 1981. Analyse factorielle des inventaires de régénération après coupe. Unité gestion du Bas-Saint-Laurent. Min. Énergie et Ressources du Québec. (polycopié), 7 p

Overend, M., 1981. Ecosystem classification - new tool for fores ters. Canadian Forest Industries, Vol. 101 (8), p. 22 et 26.

Thompson, J. 1981. Nous avons surexploite nos ressources forestières. Allocution prononcée au $73^{\mathrm{e}}$ congrès annuel de l'Association canadienne de l'industrie du bois. In. Forêt et papier. (Mars/avril 1981), p. 6

\section{FORESTRY, FISHERIES, WILDLIFE Simon Fraser University}

The Natural Resources Management Program, a graduate department at Simon Fraser University, invites applications from Ph.D.'s who wish to be sponsored for an NSERC University Research Fellowship. This position, which is at the Assistant Professorship rank, is tenable for three years beginning in 1984, and is extendable for another two years. This appointment is dependent on NSERC approval and funding. Although the primary responsibilities will be in the area of research there will also be some teaching and administrative duties.

Applications are requested in the fields of fisheries management, wildlife management, or forestry, and applicants with postdoctoral experience will be given preference. In accordance with Canadian immigration requirements, this advertisement is directed to Canadian citizens and landed immigrants. The closing date for applications is $15 \mathrm{Sep}-$ tember 1983.

Send curriculum vitae and example publications to the address below. Also, have three referees send letters of reference directly to:

Dr. J.C. Day, Director

Natural Resources Management Program

Simon Fraser University

Burnaby, B.C. V5A 1 S6

\section{SCHOOL OF FORESTRY}

\section{RESEARCH ASSOCIATES AND POST-DOCTORAL ASSISTANTS}

Research Associateships and post-doctoral assistantships are available in the School of Forestry, in the following specializations: quantitive phytosociology, tree physiology and genetics, plant population biology and taxonomy, numerical analysis of resoruce data, digital mapping and large-scale photo sampling. Duties will include teaching and research.

A Ph.D. is preferred. Applicants with a Master's and relevant work experience will be considered. Remuneration at NSERC postdoctoral level may be supplemented depending on qualifications and availability of funds.

In accordance with Canadian immigration requirements, Canadian citizens and landed immigrants will be given preferences.

Please send curriculum vitae and the names of three references to:

Dr. A.J. Kayll, Director

School of Forestry

Lakehead University

Thunder Bay, Ontario

P7B 5E1 